\title{
Synthèse
}

Génétique et génomique

\section{L'amélioration génétique de la carpe commune (Cyprinus carpio L.)}

Marc Vandeputte ${ }^{1,2}$

${ }^{1}$ Inra

UMR1313

Génétique animale et biologie intégrative Domaine de Vilvert

78350 Jouy-en-Josas

France

$<$ marc.vandeputte@jouy.inra.fr>

2 Ifremer

Chemin de Maguelone

34250 Palavas-les-Flots

France

\begin{abstract}
Résumé
La carpe commune est la première espèce de poissons à avoir été domestiquée et l'une des espèces majeures de l'aquaculture mondiale. Cependant, malgré une somme considérable de connaissances disponible sur tous les aspects de sa génétique, il n'existe pour ainsi dire pas, à l'heure actuelle, de programmes d'amélioration génétique de cette espèce. Seul le croisement de lignées est utilisé. Les raisons en sont, d'une part, l'absence de gain produit par certaines méthodes utilisées, par ailleurs, en aquaculture (monosexage, triploïdie), et, d'autre part, la mauvaise image de la sélection, qui est difficile à mettre en ouvre dans le milieu "étang ", et pour laquelle on manquait jusqu'à présent de paramètres génétiques fiables. Même avec l'acquisition récente de ces paramètres, permise par les techniques d'assignation de parenté par marqueurs microsatellites, la question de l'amélioration de la croissance reste loin d'être évidente, la croissance étant essentiellement liée à la productivité de l'étang et à la densité de mise en charge. Nous concluons que la sélection devrait s'orienter prioritairement sur la qualité du produit, et éventuellement la résistance aux maladies, plus faciles à valoriser. La croissance est un objectif à long terme, qui n'est valorisable que si des gains importants sont cumulés. La transgenèse pourrait être une option pour cela, mais, sans parler des problèmes de biosécurité et d'acceptabilité qu'elle soulève, ses résultats actuels se révèlent décevants et peu susceptibles de débloquer la situation.
\end{abstract}

Mots clés : amélioration génétique ; aquaculture ; Cyprinus carpio ; génétique des populations ; sélection.

Thèmes : amélioration génétique ; pêche et aquaculture ; productions animales.

\section{Abstract \\ Genetic improvement of common carp (Cyprinus carpio L.)}

Common carp was the first domesticated fish species and is now a major species in worldwide aquaculture. Although research has generated a considerable body of knowledge about its genetics, genetic improvement programmes are virtually non-existent in this species and only crossbreeding is used in practice. The main reasons are the inefficiency of methods usually effective in other aquaculture species (monosexing, triploidy), and the bad image of selective breeding which is difficult to implement in ponds, and for which reliable genetic parameters were missing until recently. These parameters are now becoming available through the use of parentage assignment with microsatellite markers. However, the question of selective breeding for growth remains far from simple, as growth is essentially linked to the productivity of the pond and to the stocking density of fish. We conclude that breeding should focus primarily on quality traits and possibly disease resistance which are easier to assess. Growth is a longterm objective which can only be evaluated with large cumulated gains. Transgenesis could be an option for this goal, but along with the biosafety and acceptability issues it raises, its present results are quite disappointing, and therefore this technique does not at present seem to be an immediate answer to the situation.

Key words: aquaculture; breeding; Cyprinus carpio; population genetics; selection.

Subjects: animal productions; fishing and aquaculture; genetic improvement.

Tirés à part: M. Vandeputte 
a carpe commune est une espèce majeure de l'aquaculture, avec une production de trois millions de tonnes en 2005 (FAO, 2007), mais est également le poisson le plus anciennement domestiqué (Balon, 1995). De nombreuses souches d'élevages sont disponibles, mais pour autant aucun programme de sélection suivi n'existe sur cette espèce (Hulata, 1995), alors que la sélection sur la croissance est connue pour générer des gains importants (4-20\% par génération) chez les poissons (Olesen et al., 2003).

Dans cet article, nous allons faire un tour d'horizon des connaissances sur les différentes populations de carpe, des méthodes connues et appliquées en génétique de la carpe, pour terminer par les perspectives que pourrait offrir une sélection de cette espèce sur divers critères d'intérêt économique. Pour plus de détails, on pourra se reporter à plusieurs synthèses en anglais qui ont traité de ces sujets: généralités sur la génétique de la carpe (Kirpichnikov, 1999), croisements et hybridation (Hulata, 1995), variabilité génétique et sélection (Vandeputte, 2003), manipulations chromosomiques et contrôle du sexe (Gomelsky, 2003) et transgenèse (Wu et al., 2003).

\section{Domestication}

\section{et structure des populations}

La carpe est le poisson le plus anciennement domestiqué, originaire d'Europe centrale (delta du Danube en particulier), où son élevage a été instauré par les Romains, il y a environ 2000 ans, pour se répandre et se développer ensuite durant le Moyen Âge (Balon, 2004). Son aire naturelle de répartition s'étend, cependant, jusqu'en Asie du Sud-Est, où il semble probable qu'un second événement indépendant de domestication ait eu lieu (Kohlmann et al., 2005). De nos jours, il n'existerait plus, au moins en Europe, de véritables populations naturelles, et l'on pense que toutes les carpes "sauvages" sont en réalité des échappées d'élevage revenues à l'état sauvage (Balon, 1995). Initialement, la limite ouest de l'aire de répartition serait le bassin du Danube, et donc toutes les populations "naturelles " d'Europe occidentale seraient, quoi qu'il en soit, d'origine domestique. Les études de génétique des populations montrent qu'il existe deux sous-espèces Cyprinus carpio carpio en Europe, et Cyprinus carpio haematopterus en Asie (Paaver et Gross, 1990 ; Kohlmann et Kersten, 1999; Zhou et al., 2004 ; Kohlmann et al., 2005). Parmi les autres sous-espèces parfois décrites, C. c. aralensis, est phylogénétiquement très proche de C. c. carpio (Kohlmann et al., 2005), et C. c. rubrofuscus est proche, quoique différente, de C. c. haematopterus (Zhou et al., 2004). À l'intérieur de chaque sousespèce, les distances génétiques entre populations sont faibles (Kohlmann et Kersten, 1999; Desvignes et al., 2001). On remarque également, sans surprise, que la variabilité génétique intrapopulation est plus faible chez les souches d'élevage que chez les populations "sauvages " (4,4 allèles/microsatellite contre 8,2, Kohlmann et al., 2005).

\section{Des souches \\ très diverses}

Au cours de sa longue histoire de domestication, la carpe a fait l'objet de sélections volontaires ou non, qui ont abouti à des souches très différentes, à la fois par la forme, l'écaillure, la couleur et les performances. Une des différences les plus importantes concerne l'écaillure, dont les quatre types (écaillé, miroir, linéaire, cuir) sont déterminés par deux loci indépendants $\mathrm{S}$ (scaly = écaillé) et $\mathrm{N}$ (nude = nu). Les génotypes et les phénotypes correspondants sont donnés dans le tableau 1. L'allèle $\mathrm{N}$ à l'état homozygote est létal (mortalité à l'éclosion), ce qui diminue la viabilité des populations qui le portent. Même à l'état hétérozygote $\mathrm{N} / \mathrm{n}$, des effets pléiotropiques ont été démontrés, provoquant une viabilité moindre et des déformations des nageoires chez les carpes "cuir " et "linéaires " (Kirpichnikov, 1999). Pour cette raison, l'allèle $\mathrm{N}$ a été éliminé des souches d'élevage qui sont toutes soit écaillées, soit miroir. À côté de cela, divers gènes de coloration sont connus, et sont exploités dans la constitution de souches ornementales, de type " koï " (Kirpichnikov, 1999 ; David et al., 2004).

Les souches existantes et leurs croisements ont fait l'objet de nombreuses évaluations de performances, principalement pour la croissance et la survie. La plupart de ces études utilisent des moyens de maîtrise des effets "étang ", sans lesquels les comparaisons sont très difficiles à interpréter, car les variations de performance observées entre deux étangs traités de manière identique peuvent être très élevées : une variation de croissance de 50 \% d'un étang à l'autre n'a rien d'exceptionnel. Les meilleurs moyens de contrôle de l'effet étang sont le testage communal (Wohlfarth et Moav, 1985), où les génotypes sont tous élevés en mélange, ou l'utilisation d'un témoin interne (Gross et Wohlfarth, 1994; Vandeputte et al., 2002). Les différences entre souches sont variables selon les expérimentations, mais peuvent parfois être spectaculaires entre la plus mauvaise et la meilleure d'entre elles (1:1,3 à 1:2,0 en croissance - Moav et al., 1975; Pokorný et al., 1983 ; Wohlfarth, 1993 ; Linhart et al., 2002 ; 1:1,3 à 1:2,1 en survie - Bakos et Gorda, 1995 ; Bialowas et al., 1997 ; Duda et al., 1999). Occasionnellement, des différences entre souches ont été mises en évidence sur d'autres caractères : résistance au Koï herpèsvirus (KHV) (Shapira et al., 2005), à la bactérie Aeromonas salmonicida (Wiegertjes et al., 1995), niveau d'anticorps naturels (Kachamakova et al., 2006), capturabilité à la senne (Wohlfarth et al., 1975). Par ailleurs, par l'étude de croisements, on a mis en évidence de l'hétérosis (performance de l'hybride supérieure à la moyenne des performances des lignées parentales), sur la croissance (Bialowas, 1991 ; Wohlfarth, 1993 ; Bakos et Gorda, 1995 ; Bialowas et al., 1997) et sur la survie (Bakos et Gorda, 1995; Bialowas et al., 1997). Dans ces études, l'hétérosis peut représenter 20 voire $40 \%$ de la moyenne du caractère. Desétudes plus récentes sur des souches chinoises montrent que la part des effets additifs (les effets directs des souches parentales) et non additifs (l'hétérosis) sur la croissance peut être très variable au cours du développement du poisson (Wang et al., 2006). Ces gains liés aux croisements, alliés au fait que l'absence de maitrise de la consanguinité dans les lignées de base n'est pas susceptible de faire baisser l'hétérosis, expliquent en grande partie le succès des méthodes de croisement de souches dans l'amélioration génétique de la carpe.

Dans certains essais, les mêmes souches ont été testées dans différents environnements, montrant la présence d'interactions génotype-milieu importantes, puisqu'elles peuvent aboutir à des reclas- 


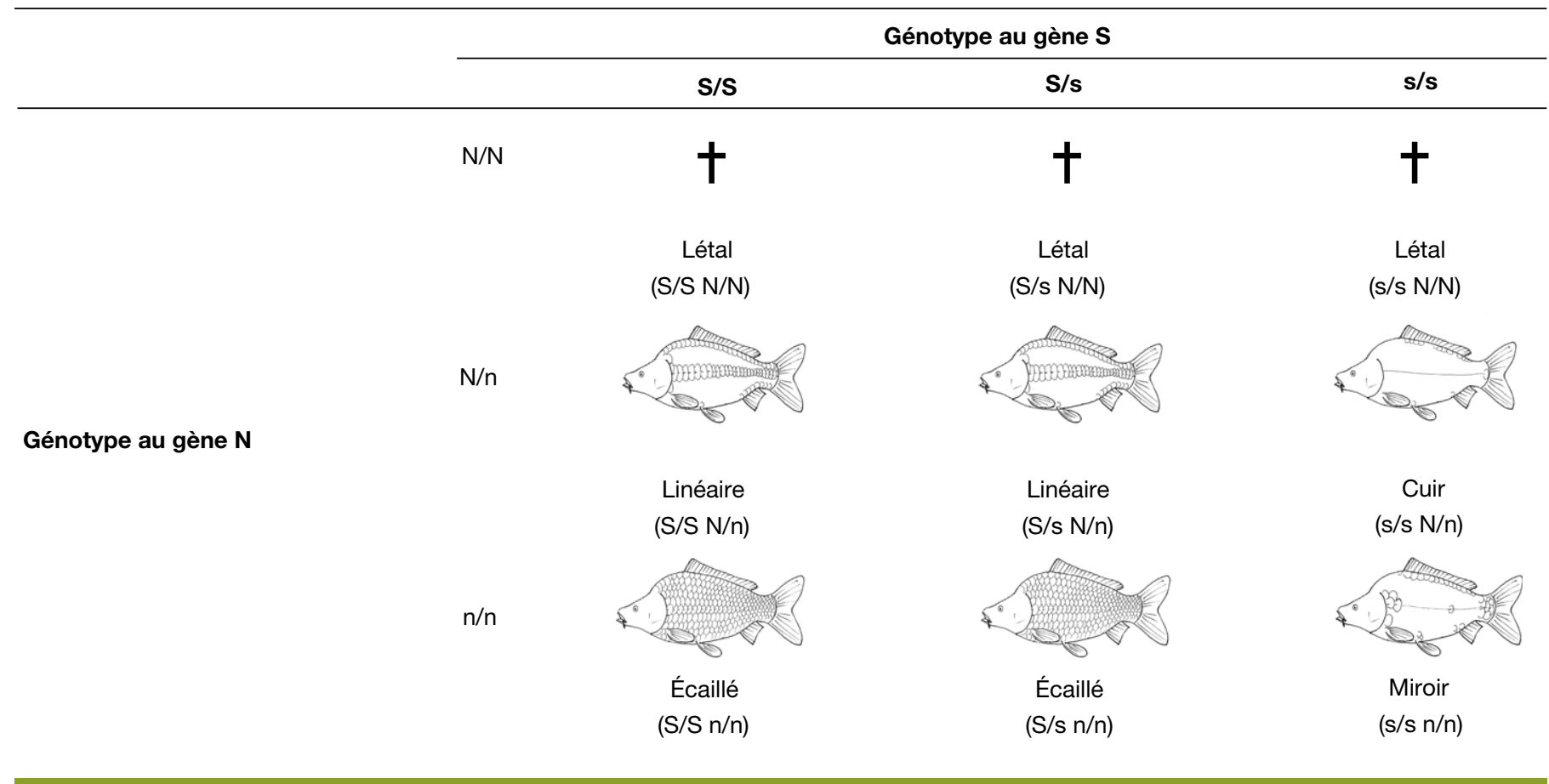

Tableau 1. Génotypes et phénotypes pour les différentes combinaisons alléliques des gènes d'écaillure de la carpe, Nu (N/n) et Scaly (S/s) (d'après Kirpichnikov, 1981).

Table 1. Genotypes and phenotypes for the different allelic combinations of scale cover genes in common carp, Nude (N/n) and Scaly (S/s) (from Kirpichnikov, 1981).

sements de la valeur relative des souches (Moav et al., 1975; Wohlfarth, 1983; Gross et Wohlfarth, 1994).

\section{Paramètres génétiques}

Pour envisager une sélection génétique intrasouche, il est nécessaire d'évaluer les paramètres génétiques des caractères, et au premier chef leur héritabilité. De nombreuses estimations ont été faites dans les années 1960-1980 et jusqu'à 2000, sur des caractères de croissance (longueur, poids) et de forme, mais aussi sur la résistance à l'hypoxie, le poids de gonades, le taux de larves déformées ou le contenu en lipides (Vandeputte, 2003). Ces estimations ont été obtenues dans des dispositifs de taille très limitée avec une phase d'élevage initial (avant le marquage) en familles séparées, susceptible de biaiser fortement les estimations. Elles sont globalement compatibles avec une héritabilité inférieure ou égale à 0,30 pour la croissance, et sans doute un peu plus élevée $(0,30-0,40)$ pour la conformation (rapport hauteur/ longueur). La mise au point de marqueurs microsatellites chez la carpe (Crooijmans et al., 1997 ; David et al., 2001) a autorisé le développement de l'assignation de parenté par génotypage, qui permet d'avoir des estimations de paramètres génétiques beaucoup plus fiables, utilisant de nombreuses familles élevées dans un étang unique. Ces études confirment une héritabilité de la croissance de l'ordre de 0,20 à 0,40 (Vandeputte et al., 2004 ; Vandeputte et al., 2008) et de 0,30 à 0,40 pour le coefficient de condition (poids/longueur au cube). Des corrélations génétiques assez faibles $(0,35$ à $0,70)$ entre le même critère de croissance (longueur ou poids), mesuré dans des conditions différentes, pourraient indiquer l'existence d'interactions génotypemilieu au niveau familial, ce qui pourrait compliquer la sélection de lignées à croissance rapide (Vandeputte et al., 2008).

L'héritabilité des caractères de qualité est assez élevée : 0,58 pour le gras musculaire, 0,28 pour le rendement en carcasse et 0,38 pour le rendement en filet, ce dernier pouvant être prédit efficacement par la longueur relative de la tête, avec une corrélation génétique de -0,86 (Kocour et al., 2007). La réponse de stress à un choc froid (mesurée par le taux de cortisol plasmatique) est, elle aussi, fortement héritable ( $\mathrm{h}^{2}=0,60$, Tanck et al., 2001b). Enfin, les malformations de la bouche ne seraient pas héritables, alors que les malformations des nageoires pourraient avoir une base génétique limitée (Kocour et al., 2006). Pour ce qui est des corrélations génétiques, la forme et la croissance semblent assez peu corrélées en général (Ankorion et al., 1992 ; Vandeputte et al., 2004 ; Kocour et al., 2007), ce qui laisse la possibilité de les sélectionner de façon assez indépendante. La croissance et le taux de gras musculaire sont assez fortement corrélés (de $+0,60$ à $+0,70)$, ce qui est plutôt défavorable, mais à l'inverse, la corrélation génétique entre croissance et rendement en filet (de $+0,50$ à $+0,60)$ est favorable (Kocour et al., 2007). Il faut noter que, depuis 2004, une carte génétique de la carpe comportant 272 marqueurs, dont 110 microsatellites, est disponible (Sun et Liang, 2004). Elle a permis de localiser un locus associé à la tolérance au froid. D'autres associations marqueurscaractères avaient été mises en évidence en 2001 sur la croissance et la tolérance au stress (Tanck et al., 2001a), mais rien n'a été publié depuis 2004. 


\section{Réponse à la sélection}

Si la sélection de la carpe a été pratiquée de façon empirique depuis des siècles, une pratique un peu plus rationnelle a vu le jour au début du $\mathrm{Xx}^{\mathrm{e}}$ siècle (Drouin de Bouville, 1930), mais les expériences de sélection bien documentées sont rares. La plus complète est celle menée en Israël dans les années 1970, qui portait sur la première année de croissance. Elle s'est poursuivie sur cinq générations de sélection divergente (une lignée à croissance augmentée, une lignée à croissance diminuée), pour aboutir à un échec : la sélection montante montra un succès mitigé sur la première génération, pour s'écrouler sur les deux générations suivantes, et se stabiliser au cours des quatrièmes et cinquièmes générations, bien en deçà du niveau du témoin (Moav et Wohlfarth, 1976). La sélection descendante, quant à elle, fut efficace sur les trois premières générations, et se stabilisa ensuite au cours des deux suivantes. Les auteurs en conclurent que la carpe était arrivée à un plateau de sélection pour la croissance, du fait de la sélection empirique pratiquée depuis des siècles. Il nous semble plus probable que la méthode de reproduction employée (pontes en masse) soit à l'origine d'une consanguinité élevée, car il est peu probable, dans ce cas, que tous les parents potentiels participent à la ponte. Cette technique ne garantit pas non plus une ponte absolument synchrone, ce qui peut amener à favoriser les descendants des pontes les plus précoces au détriment de ceux qui ont la meilleure capacité de croissance (Wohlfarth et Moav, 1970). Une autre expérience de sélection sur la croissance réalisée au Vietnam annonce une héritabilité réalisée de 0,20 à 0,29, mais sans donner de détails permettant d'évaluer les résultats (Tran et Nguyen, 1993). Enfin, une étude récente sur une génération montre également une héritabilité réalisée assez importante pour la sélection montante (0,24-0,34), mais nulle pour la sélection descendante (Vandeputte et al., 2008). Au final, il semble raisonnable de compter sur une héritabilité réalisée de 0,20 à 0,30 de la croissance, et donc sur une possibilité de sélectionner ce caractère chez la carpe. Par ailleurs, une expérience de sélection sur la forme (ratio hauteur/longueur) a monté une réponse favorable de ce caractère, vers le haut comme vers le bas, et dans des proportions compatibles avec les estimations d'héritabilité existantes (Ankorion et al., 1992). Enfin, deux expériences de sélection pour la résistance à l'hydropisie ont été entreprises avec succès (Schäperclaus, 1962 ; Kirpichnikov et al., 1993; Kirpichnikov, 1999 - mais l'hydropisie étant un symptôme, on peut se demander quel est le pathogène associé), ainsi que la sélection de la carpe " Ropsha " résistante aux froids hivernaux (Kirpichnikov et al., 1974 ; Babouchkine, 1987). Au final, il faut cependant constater qu'aujourd'hui, aucun programme commercial de sélection de carpe commune n'est en place à notre connaissance, malgré ces divers résultats plutôt encourageants.

\section{Manipulations des stocks chromosomiques} et monosexage

Ces techniques, largement utilisées chez les salmonidés, et en particulier chez la truite arc-en-ciel (Haffray et al., 2004), sont maîtrisées chez la carpe. Le déterminisme du sexe chez la carpe étant de type $\mathrm{XX}-\mathrm{XY}$, le monosexage femelle est obtenu initialement par gynogenèse (Komen et al., 1991), puis des "néomâles " sont produits par inversion sexuelle hormonale (17 $\alpha$-méthyltestostérone dans l'aliment) de ces femelles génétiques (Gomelsky et al., 1995). Ces néomâles (XX), par croisement sur des femelles ordinaires $(\mathrm{XX})$ ne produisent que des animaux XX, donc femelles. Les essais de comparaison en étangs de populations monosexes femelles avec des populations bisexuées montrent un avantage de poids pour les populations monosexes à un et deux ans, qui disparaît à taille commerciale (trois ou quatre ans : Kocour et al., 2005). Cette méthode, bien que maitrisée, ne présente donc pas de potentiel d'application.

L'induction de la triploïdie, qui permet une stérilisation génétique des animaux, et en conséquence permet d'espérer des gains de croissance somatique, est également maîtrisée chez la carpe. Les mises au point ont eu lieu au cours des années 1980-1990 (Gomelsky, 2003), pour aboutir à des méthodes efficaces (80-100\% de triploïdes) et applicables en écloserie (Recoubratsky et al., 1992). Cependant, en conditions d'élevage, les triploïdes ont une survie et une croissance plus fai- bles que les diploïdes ( -30 et $-15 \%$ respectivement, Cherfas et al., 1994). Ils n'ont donc jamais été utilisés en élevage, et ont un potentiel très faible, la question de la stérilisation pour éviter la contamination des stocks sauvages ne se posant pas chez la carpe (au moins en Europe), sauf dans l'hypothèse éventuelle d'élevage de transgéniques.

\section{Transgenèse}

Comme plusieurs espèces de poissons d'aquaculture, la carpe a fait l'objet d'essais de transfert de gènes d'hormone de croissance (Zhang et al., 1990 ; Zhu, 1992 ; Moav et al., 1995). Les différents auteurs annoncent des gains de croissance plus ou moins importants ; cependant, les conditions expérimentales ne sont jamais idéales quand des gains majeurs sont avancés :

- Fu et al., 1998 annoncent un gain de taux de croissance journalier d'environ $20 \%$ (soit un gain de poids supérieur de $50 \%$ sur huit semaines), mais le test est conduit sur une période courte, en aquariums, et en comparant des transgéniques de quatrième génération avec des témoins dont on sait juste qu'ils sont de la même variété (carpe rouge) ;

- Fu et al., 2007 montrent un taux de croissance journalier supérieur de $70 \%$ (soit un gain de poids double sur 21 jours), mais avec des effectifs très faibles (dix transgéniques, dix non transgéniques), un élevage en aquariums individuels (un poisson/aquarium), une expérience très courte et un témoin d'origine non précisée.

Dans un test en étangs sur quatre mois aux États-Unis, en comparant dans les mêmes étangs des transgéniques F1 ou F2 avec leurs frères et sœurs non transgéniques, on voit des gains beaucoup plus modestes, de 0 à $49 \%$ de poids en plus chez les transgéniques selon les familles, avec une moyenne de $17 \%$ environ (Dunham et al., 2002). Dans un autre test de croissance en étangs en Israël, on a pu montrer une absence de supériorité des transgéniques en été (période à croissance forte), mais un gain significatif (taux de croissance $\times 2$ ) en hiver, période de faible croissance (Hinits et Moav, 1999). En Hongrie, des tests en étangs ont été annoncés en 1995 (Horvath et Orban, 1995), mais aucune donnée publiée n'a suivi cette annonce. En conclusion, les 
performances prouvées des carpes transgéniques GH sont pour l'instant modestes, ce qui n'est pas nécessairement surprenant: les carpes sont domestiquées depuis des siècles, et chez la truite arcen-ciel, il a été montré que les souches sauvages répondaient très favorablement à la transgenèse $\mathrm{GH}$, alors que les mêmes constructions testées sur des truites de souche domestique ne produisaient aucun gain de croissance (Devlin et al., 2001). Pour ce qui est de l'application en production de la transgenèse, certains scientifiques chinois considèrent que la faisabilité technique est prouvée, et que l'évaluation des risques a été faite (Wu et al., 2003). Cependant, l'administration n'a pas encore autorisé leur usage et demande une évaluation complète des risques associés, incluant une reconnaissance et une prise en compte des inquiétudes du public à leur sujet (Fu et al., 2005). Aucune carpe transgénique n'est donc actuellement élevée hors des centres de recherche en Chine ( $\mathrm{Fu}$ et al., 2005). Dans les autres pays (États-Unis, Israël et Hongrie), rien n'indique que le développement de lignées de carpes transgéniques soit toujours d'actualité.

\section{Perspectives}

Si les connaissances sur la génétique de la carpe, tant pour ce qui est de la diversité des populations, des performances des souches, des paramètres génétiques, des manipulations des chromosomes, du sexe et du génome, représentent un corpus important, acquis essentiellement depuis les années 1960, force est de constater que ces résultats sont bien peu utilisés en pratique, mis à part l'utilisation de souches préalablement caractérisées et de leurs croisements, essentiellement en Europe centrale et depuis peu en Chine. Les raisons sont à rechercher à plusieurs niveaux :

- certaines méthodes, utilisées chez d'autres espèces (monosexage, triplö̈die) semblent inefficaces chez la carpe ;

- la difficulté de suivi des généalogies a induit un manque, jusqu'à une époque récente, de paramètres génétiques fiables. L'échec connu de tous du programme de sélection israélien a détourné la recherche de la sélection chez cette espèce. Cependant, la possibilité d'utiliser des assignations de parenté par marqueurs génétiques a permis d'obtenir des résultats nouveaux, qui offrent des perspectives plus favorables à la mise en place de schémas de sélection. Si le coût de ces méthodes (10-15 euros/poisson) est encore un obstacle en 2007, leur réduction est envisageable, par exemple, par le développement de marqueurs de type SNP (single nucleotide polymorphism) ;

- l'élevage de la carpe est resté pour l'essentiel une activité familiale ou de petites et moyennes entreprises, à l'inverse de l'aquaculture des salmonidés ou de la crevette, où de grandes entreprises ont les moyens d'investir dans des programmes lourds de sélection.

Enfin, et ce n'est pas le moindre problème, la carpe étant essentiellement élevée en étangs, à partir de la productivité naturelle du milieu, éventuellement complémentée par une alimentation exogène, la croissance des individus est essentiellement modulée par la quantité de nourriture disponible dans le milieu, la densité de mise en charge et la mortalité (Szumiec, 1990). Il est loin d'être évident que des animaux à croissance plus rapide puissent augmenter la productivité totale de l'étang, et donc leur utilisation nécessiterait vraisemblablement une adaptation des pratiques (réduction des densités de mise en charge), difficile à justifier et à mettre en ouvre tant que le génotype sélectionné n'aura pas une croissance nettement supérieure aux souches habituelles, ce qui pourrait prendre plusieurs dizaines d'années au rythme prévisible d'amélioration génétique de la croissance. La transgenèse pour la croissance, qui pour l'instant n'a pas montré son efficacité pratique chez la carpe, poserait d'ailleurs les mêmes questions. Il semble donc que l'avenir de l'amélioration génétique de la carpe soit une amélioration de la qualité (rendements de chair, lipides corporels, forme) ou de la résistance à certains pathogènes (on pense au KHV, maladie nouvelle ayant d'importantes conséquences - Haenen et al., 2004) dont le bénéfice pourrait être plus immédiat. Un programme de sélection intégrant ces critères pourrait en même temps permettre une certaine pression de sélection pour l'amélioration de la croissance pour le plus long terme, tout en trouvant une valorisation plus immédiate. Enfin, techniquement, il ne faut pas négliger la proximité génétique de la carpe et du poisson zèbre, qui pourrait permettre d'accéder assez rapidement à une excellente connaissance du génome de la carpe, ouvrant de nouvelles voies pour l'amélioration génétique (Christoffels et al., 2006).

\section{Références}

Ankorion Y, Moav R, Wohlfarth GW. Bidirectionnal mass selection for body shape in common carp. Genet Sel Evol 1992 ; 24 : 43-52.

Babouchkine YP. La sélection d'une carpe résistant à I'hiver. In : Tiews $\mathrm{K}$, ed. Proceedings of the world symposium on selection, hybridization, and genetic engineering in aquaculture. Berlin: Heenemann Verlagsgesellschaft $\mathrm{mbH}$, 1987

Bakos J, Gorda S. Genetic improvement of common carp strains using intraspecific hybridization. Aquaculture 1995 ; 129 : 183-6.

Balon EK. About the oldest domesticates among fishes. J Fish Biol 2004 ; 65 : 1-27.

Balon EK. Origin and domestication of the wild carp, Cyprinus carpio: from Roman gourmets to the swimming flowers. Aquaculture 1995 $129: 3-48$.

Bialowas $\mathrm{H}$. Possibilities of application of the heterosis effect in commercial production of common carp (Cyprinus carpio L.). 1. Production of fingerlings. Acta Hydrobiol 1991; 33 : 319-34.

Bialowas H, Irnazarow I, Pruszynski T, Gaj C. The effect of heterosis in interline crossing of common carp. Arch Ryb Pol 1997 ; 5 : 13-20.

Cherfas NB, Gomelsky B, Ben-Dom N, Peretz Y, Hulata G. Assessment of triploid common carp (Cyprinus carpio L.) for culture. Aquaculture $1994: 127: 11-8$

Christoffels A, Bartfai R, Srinivasan H, Komen $H$, Orban L. Comparative genomics in cyprinids: common carp ESTs help the annotation of the zebrafish genome. BMB Bioinformatics 2006 ; S5 : S2.

Crooijmans RPMA, Bierbooms VAF, Komen J, Van der Poel JJ, Groenen MAM. Microsatellite markers in common carp (Cyprinus carpio L.). Anim Genet $1997 ; 28: 129-34$.

David L, Rajasekaran P, Fang J, Hillel J, Lavi U. Polymorphism in ornamental and common carp strains (Cyprinus carpio L.) as revealed by AFLP analysis and a new set of microsatellite markers. Mol Genet Genomics 2001; 266 : 353-62.

David L, Rothbard S, Rubinstein I, et al. Aspects of red and black color inheritance in the Japanese ornamental (Koï) carp (Cyprinus carpio L.). Aquaculture 2004 ; 233 : 129-47.

Desvignes JF, Laroche J, Durand JD, Bouvet $Y$. Genetic variability in reared stocks of common carp (Cyprinus carpio L.) based on allozymes and microsatellites. Aquaculture 2001; 194 : 291-301.

Devlin RH, Biagi CA, Yesaki TY, Smailus DE, Byatt JC. Growth of domesticated transgenic fish. Nature $2001 ; 409$ : 781-2.

Drouin de Bouville R. Une réussite carpicole : le croisement Beauvoir ${ }^{\circ}$ 1. B Fr Piscic 1930 $20: 169-76$.

Duda P, Gela D, Linhart O. Top-crossing with paternal inheritance testing of 4-month-old common carp Cyprinus carpio L. progeny in three altitude conditions. Aquacult Res 1999 ; $30: 911-6$ 
Dunham RA, Chatakondi N, Nichols AJ, et al. Effect of rainbow trout growth hormone complementary DNA on body shape, carcass yield, and carcass composition of F1 and F2 transgenic common carp (Cyprinus carpio). Mar Biotechnol 2002 ; 4 : 604-11.

FAO. Aquaculture production: quantities 19502005. FISHSTAT Plus-Available at: http://www. fao.org/fi/statist/FISOFT/FISHPLUS.as, 2007.

Fu C, Cui Y, Hung SSO, Zhu Z. Growth and feed utilization by $\mathrm{F} 4$ human growth hormone transgenic carp fed diets with different protein levels. J Fish Biol 1998; 53 : 115-29.

Fu C, Hu W, Wang Y, Zhu Z. Developments in transgenic fish in the People's Republic of China. Rev Sci Tech Off Int Epiz 2005; 24 : 299-307.

Fu C, Li D, Hu W, Wang Y, Zhu Z. Growth and energy budget of F2 'all-fish' growth hormone gene transgenic common carp. J Fish Biol 2007 ; 70 : 347-61.

Gomelsky B. Chromosome set manipulation and sex control in common carp: a review. Aquat Living Resour 2003 ; 16 : 408-15.

Gomelsky BI, Cherfas NB, Hulata G, Peretz Y, Ben-Dom N. Hormonal sex inversion in the common carp, Cyprinus carpio L. Aquaculture $1995 ; 129: 218$.

Gross R, Wohlfarth GW. Use of genetic markers in growth testing of common carp, Cyprinus carpio L., carried out over two or three years cycles. Aquacult Fish Manage 1994; 25 : 585-99.

Haenen OLM, Way K, Bergmann SM, Ariel E. The emergence of koï herpes virus and its significance to European aquaculture. Bull Eur Assn Fish P 2004 ; 24 : 293-307.

Haffray P, Pincent C, Rault P, Coudurier B. Domestication et amélioration génétique des cheptels piscicoles francais dans le cadre du SYSAAF. INRA Prod Anim 2004; 17: 243-52.

Hinits $Y$, Moav B. Growth performance studies in transgenic Cyprinus carpio. Aquaculture $1999 ; 173: 285-96$.

Horvath L, Orban L. Genome and gene manipulation in the common carp. Aquaculture $1995 ; 129$ : 157-81.

Hulata G. A review of genetic improvement of the common carp (Cyprinus carpio L.) and other cyprinids by crossbreeding, hybridization and selection. Aquaculture 1995; 129: 143-55.

Kachamakova NM, Irnazarow I, Parmentier HK, Savelkoul HFJ, Pilarczyk A, Wiegertjes GF. Genetic differences in natural antibody levels in common carp (Cyprinus carpio L.). Fish Shellfish Immunol 2006; 21 : 404-13.

Kirpichnikov VS. Genetics and breeding of common carp. Paris : Inra éditions, 1999.

Kirpichnikov VS. Genetic bases of fish selection. Berlin : Springer-Verlag, 1981.

Kirpichnikov VS, Ilyasov I, Shart LA, et al. Selection of Krasnodar common carp (Cyprinus carpio) for resistance to dropsy: principal results and prospects. Aquaculture 1993; 111 : 7-20.

Kirpichnikov VS, Ponomarenko KV, Tolmacheva NV, Tsoi RM. Methods and effectiveness of breeding Ropshian carp. II. Methods of selection. Sov Genet $1974 ; 10$ : 1108-16.

Kocour M, Linhart O, Gela D, Rodina M. Growth performance of all-female and mixedsex common carp Cyprinus Carpio L. Populations in the Central Europe climatic conditions. J World Aquacult Soc 2005 ; 36 : 103-13.
Kocour M, Linhart $\mathrm{O}$, Vandeputte M. Mouth and fin deformities in common carp: is there a genetic basis? Aquacult Res 2006; 37 419-22.

Kocour M, Mauger S, Rodina M, Gela D, Linhart $\mathrm{O}$, Vandeputte M. Heritability estimates for processing and quality traits in common carp (Cyprinus carpio L.) using a molecular pedigree. Aquaculture $2007 ; 270$ : 43-50.

KohImann K, Kersten P. Genetic variability of German and foreign common carp (Cyprinus carpio L.) populations. Aquaculture 1999; $173: 435-45$.

Kohlmann K, Kersten P, Flajshans $M$ Microsatellite-based genetic variability and differentiation of domesticated, wild and feral common carp (Cyprinus carpio L.) populations. Aquaculture $2005 ; 247: 253-66$.

Komen J, Bongers ABJ, Richter CJJ, Van Muiswinkel WB, Huisman EA. Gynogenesis in common carp (Cyprinus carpio L.): II. The production of homozygous gynogenetic clones and F1 hybrids. Aquaculture 1991; 92 : 127-42.

Linhart O, Gela D, Rodina M, Slechtova V, Slechta V. Top-crossing with paternal inheritance testing of common carp (Cyprinus carpio L.) progeny under two altitude conditions. Aquaculture $2002 ; 204$ : 481-91.

Moav B, Hinits Y, Groll Y, Rothbard S. Inheritance of recombinant carp [beta]-actin/GH cDNA gene in transgenic carp. Aquaculture $1995 ; 137: 179-85$.

Moav R, Hulata G, Wohlfarth GW. Genetic differences between the Chinese and European races of the common carp I: analysis of genotype-environment interactions for growth rate. Heredity $1975 ; 34: 323-40$.

Moav R, Wohlfarth GW. Two ways selection for growth rate in the common carp (Cyprinus carpio L.). Genetics 1976 ; 82 : 83-101.

Olesen I, Gjedrem T, Bentsen HB, Gjerde B, Rye $M$. Breeding programmes for sustainable aquaculture. J Appl Aquacult 2003; 13: 179-204.

Paaver T, Gross R. Genetic variability of carp (Cyprinus carpio) strains reared in Estonia. Genetica (Moscow) 1990 ; 26 : 1269-78.

Pokorný J, Hartvich P, Ryšavý J, Klézl L. Hodnocení hmotnosti kapřiho plùdku K1 $\mathrm{s}$ použitím standardní linie. Zivocisna Vyroba 1983; $28: 843-9$.

Recoubratsky AV, Gomelsky BI, Emelyanova OV, Pankratyeva EV. Triploid common carp produced by heat shock with industrial fishfarm technology. Aquaculture 1992; 108 : 13-9.

Schäperclaus W. Traité de pisciculture en étang. Paris : Vigot Frères, 1962.

Shapira Y, Magen Y, Zak T, Kotler M, Hulata G, Levavi-Sivan B. Differential resistance to ko herpes virus (KHV)/carp interstitial nephritis and gill necrosis virus (CNGV) among common carp (Cyprinus carpio L.) strains and crossbreds. Aquaculture 2005 ; 245 : 1-11.

Sun $X$, Liang L. A genetic linkage map of common carp (Cyprinus carpio L.) and mapping of a locus associated with cold tolerance. Aquaculture $2004 ; 238$ : 165-72.

Szumiec MA. Stochastic model of carp fingeling growth. Aquaculture $1990 ; 91$ : 87-99.

Tanck MWT, Palstra AP, Van de Weerd M, et al. Segregation of microsatellite alleles and residual heterozygosity at single loci in homozygous androgenetic common carp (Cyprinus carpio L.). Genome 2001 ; 44 : 743-51.
Tanck MWT, Vermeulen $H$, Bovenhuis $H$, Komen J. Heredity of stress-related cortisol response in androgenetic common carp (Cyprinus carpio L.). Aquaculture 2001; 199: 283-94.

Tran MT, Nguyen CT. Selection of common carp (Cyprinus carpio L.) in Vietnam. Aquaculture $1993 ; 111: 301-2$.

Vandeputte M. Selective breeding of quantitative traits in the common carp (Cyprinus carpio L.): a review. Aquat Living Resour 2003; 16 : 399-407.

Vandeputte M, Kocour M, Mauger S, et al. Heritability estimates for growth-related traits using microsatellite parentage assignment in juvenile common carp (Cyprinus carpio L.). Aquaculture 2004 ; 235 : 223-36.

Vandeputte $\mathrm{M}$, Kocour $\mathrm{M}$, Mauger $\mathrm{S}$, et al. Genetic variation for growth at one and two summers of age in the common carp (Cyprinus carpio L.): heritability estimates and response to selection. Aquaculture 2008; 277: $7-13$.

Vandeputte M, Peignon E, Vallod D, Haffray P, Komen J, Chevassus B. Comparison of growth performances of three French strains of common carp (Cyprinus carpio) using hemiisogenic scaly carp as internal control. Aquaculture $2002 ; 205: 19-36$.

Wang CH, Li SF, Liu ZG, et al. Developmental quantitative genetic analysis of body weight and morphological traits in red common carp, Cyprinus carpio L. Aquaculture 2006; 251: 219-30.

Wiegertjes GF, Pilarczyk A, Van Muiswinkel WB. Disease resistance and growth of two inbred carp Cyprinus carpio L. lines and their hybrid in pond culture. Aquacult Res $1995 ; 26$ : 797-800.

Wohlfarth G, Moav R, Hulata G, Beiles A. Genetic variation in seine escapability of the common carp. Aquaculture $1975 ; 5$ : 375-87.

Wohlfarth GW. A genotype $X$ environment interaction for growth rate in the common carp, growing in intensively manured ponds. Aquaculture 1983 ; 33 : 187-95 (187-195).

Wohlfarth GW. Heterosis for growth rate in common carp. Aquaculture 1993; 113 : 31-46.

Wohlfarth GW, Moav R. Communal testing, a method of testing growth rate of different genetic groups of common carp in earthen ponds. Aquaculture $1985 ; 48$ : 143-57.

Wohlfarth GW, Moav R. The effects of variation in spawning time on subsequent relative growth rate and viability in carp. Bamidgeh $1970 ; 22: 42-7$.

Wu G, Sun Y, Zhu Z. Growth hormone gene transfer in common carp. Aquat Living Resour $2003 ; 16: 416-20$

Zhang P, Hayat M, Joyce C, et al. Gene transfer, expression and inheritance of pRSVrainbow trout-GH cDNA in the common carp, Cyprinus carpio (Linnaeus). Mol Reprod Dev $1990 ; 25: 3-13$.

Zhou J, Wu O, Wang Z, Ye Y. Molecular phylogeny of three subspecies of common carp Cyprinus carpio, based on sequence analysis of cytochrome $b$ and control region of mtDNA. J Zoological Syst Evol Res 2004; 42 : 266-9.

Zhu Z. Generation of fast growing transgenic fish: methods and mechanisms. In: Transgenic fish. Singapore: World Scientific Publishing Co. Ltd., 1992. 